\title{
Kink stability, propagation, and length-scale competition in the periodically modulated sine-Gordon equation
}

\author{
Angel Sánchez \\ Theoretical Division and Center for Nonlinear Studies, Los Alamos National Laboratory, Los Alamos, New Mexico 87545 \\ and Escuela Politécnica Superior, Universidad Carlos III de Madrid, E-28911 Leganés, Madrid, Spain \\ A. R. Bishop \\ Theoretical Division and Center for Nonlinear Studies, Los Alamos National Laboratory, Los Alamos, New Mexico 87545 \\ Francisco Domínguez-Adame \\ Departamento de Física de Materiales, Facultad de Físicas, Universidad Complutense, E-28040 Madrid, Spain
}

(Received 24 November 1993)

\begin{abstract}
We have examined the dynamical behavior of the kink solutions of the one-dimensional sineGordon equation in the presence of a spatially periodic parametric perturbation. Our study clarifies and extends the currently available knowledge on this and related nonlinear problems in four directions. First, we present the results of a numerical simulation program that are not compatible with the existence of a radiative threshold predicted by earlier calculations. Second, we carry out a perturbative calculation that helps interpret those previous predictions, enabling us to understand in depth our numerical results. Third, we apply the collective coordinate formalism to this system and demonstrate numerically that it reproduces accurately the observed kink dynamics. Fourth, we report on the occurrence of length-scale competition in this system and show how it can be understood by means of linear stability analysis. Finally, we conclude by summarizing the general physical framework that arises from our study.
\end{abstract}

PACS number(s): 03.40.Kf, 85.25.Cp, 02.90.+p

\section{INTRODUCTION}

Technological progress has made possible the fabrication of highly ordered materials and structures for a very large number of applications. In parallel to those advances, it has also been realized that the special properties required for many purposes necessitate inhomogeneous systems. Here inhomogeneity may mean spatial modulations, quasiperiodicity, or disorder of several kinds. In addition, there are other situations in which inhomogeneity is undesirable but unavoidable. In either case, the study of disordered systems acquires fundamental importance. This is even more so when the physical system in which disorder or inhomogeneity is to be studied is described by a nonlinear model. Whereas the role of disorder in linear problems is at least partially understood, much less is known about nonlinear disordered systems. In fact, even from a mathematical viewpoint, the understanding of these models, often related to stochastic partial differential equations (PDE's), is very limited. Consequently, a great deal of research has been devoted to this topic [1-3].

A major part of the work done so far regarding nonlinear disordered systems has been concerned with some particular examples that are amenable to analytical treatment while capturing some essential physics. The sine-Gordon (SG) (actually, the whole family of nonlinear Klein-Gordon equations, including, e.g., the $\phi^{4}$, double- and quadratic-sine-Gordon equations) and nonlinear Schrödinger (NLS) equations are often chosen as very suitable "canonical" examples. This is due to the fact that the basic mathematical structure underlying them is well known and therefore provides a good starting point for theoretical work. This reason would not be sufficient if these models were not also related to a large number of phenomena that occur in quasi-one-dimensional physical systems, as is in fact the case. In the context of these two models, disorder is introduced through suitably chosen perturbation terms (see [2] for an extensive list of physically relevant perturbations). This is the usual procedure by which inhomogeneity of any kind is studied: The equation describing the problem is established, the terms relevant to the considered physical situation are identified, and a perturbation to those terms is introduced, representing the desired kind of disorder. Our viewpoint in this work is more generic: Although the system we deal with is indeed related to a number of applications, our aim is that we will be able to gain insight into underlying mechanisms of the phenomenology of nonlinear disordered systems. Therefore, we introduce a simple periodic perturbation which will allow us to study very interesting and general phenomena, such as length-scale competition, and will provide information relevant to the more complicated processes occurring in random media (the periodic potential can be interpreted as a "color" of a general noisy one). The knowledge obtained will also be useful to tackle other problems where detailed studies including analytical treatment are not possible.

In this paper we study the behavior of one-dimensional (1D) SG kinks when perturbed parametrically by a spa- 
tially periodic potential. Initially, this was motivated by our related research (from the above general point of view) on the SG $[4,5]$ and NLS $[6,7]$ models. As a preliminary step to the investigation of SG breather dynamics [4] on these kind of potentials, it is natural to first seek a good understanding of kink dynamics. Therefore we undertook that study, both analytically and numerically. Our point of departure was early theoretical work [8-10] on this problem, which we summarize for completeness in Sec. II. In particular, it had been predicted that a certain critical velocity exists at which the radiative power emitted by the kink would diverge. Below that critical velocity, radiation would be zero, and above it, it would decrease with increasing speed (see Ref. [2] for a summary). Those results were obtained at a time where the main aim was to develop a perturbative approach to deal with soliton problems. That, and the fact that computers were not the easily available tool they are nowadays, meant that those results were never analyzed in depth or numerically checked. Therefore, as a first stage of our study, we devised a number of numerical experiments to check them, and we found no numerical evidence for the predicted divergence. In view of this result, we carried out an improved perturbative calculation, in the sense that it allowed us to interpret correctly the earlier results in Refs. [8-10] and to show that, although the earlier analyses were correct, the predicted divergence was actually unphysical. This theoretical analysis is reported in detail in Sec. III: A preliminary short report has been given elsewhere [11]. The work done to that point suggested to us the idea that, opposite to what was believed to date, SG kink dynamics on a periodic potential could be essentially that of a (pseudorelativistic) particle. We thus applied a simple collective coordinate formalism to the problem, and it turned out to describe soliton behavior very accurately, even predicting unexpected new phenomena. The analytical approach and the numerical simulations are contained in Sec. IV. Finally, to complete our program studying length-scale competition and its effects in nonlinear disordered systems, we performed further numerical experiments to clarify whether robust objects such as kinks, which according to our collective coordinate theory behave mostly like particles, can still exhibit the destabilizing effect of length-scale competition. We found that this was actually the case. Furthermore, the simplicity of kinks allowed us to carry out a (numerical) linear stability analysis which provided us with a clear explanation for the numerically observed features. We collect our results on this question in Sec. V. To conclude, we summarize the facts that we have learned, which considerably enhance the understanding of sineGordon kink propagation in disordered media and shed light on the so far unexplained phenomenon of lengthscale competition. Our results are also of relevance to many nonlinear systems of physical interest, mainly in three directions: First, all non-numerically validated or nonphysically interpreted predictions obtained through perturbative calculations should be treated with a degree of caution. Second, the collective coordinate formalism yields a very simple and accurate way to deal with perturbed nonlinear problems, especially those in which the perturbation enters parametrically rather than additively. And third, length-scale competition is an ubiquitous phenomenon that may be responsible for many instabilities arising in different nonlinear disordered systems.

\section{BRIEF SUMMARY OF PREVIOUS RESULTS}

We start by describing the picture of SG kink propagation on parametric periodic potentials which has been accepted to date. The problem, which has been studied by Mkrtchyan and Shmidt [8] and Malomed and Tribelsky $[9,10]$, is given by a perturbed $\mathrm{SG}$ equation of the form

$$
u_{t t}-u_{x x}+[1+\epsilon \cos (k x)] \sin u=0
$$

(modeling, for instance, a long Josephson junction with modulated critical current, to mention just one application); the question posed was whether kinks can propagate freely in such a system, and if so, to describe this propagation. We will only record here a short summary of previous work. The reader is referred to the original papers [8-10] for details.

Mkrtchyan and Shmidt [8] used a Green's function perturbation technique (GFPT). They derived a linearized equation for the first order correction to a kink moving with constant velocity, computed the Green's function corresponding to its homogeneous version, and then used it to obtain the desired correction by integrating the source term with that Green's function. They then noticed that radiation appeared only above a critical kink velocity $v_{\text {thr }}=\left(1+k^{2}\right)^{-1 / 2}$. At that particular value, the correction diverges, and the authors explain that their calculation becomes invalid in that region as, of course, it assumed the correction was small. On the other hand, the approach of Malomed and Tribelsky $[9,10]$ was quite different. Its basis was the inverse scattering perturbation theory (ISPT). A meaningful summary of this kind of calculation would be quite lengthy and hence we will omit it here, referring the reader to Ref. [2], which is mostly devoted to describing ISPT in detail. Let us just mention that the idea is that, if the amount of radiation emitted by the kink is small, as it should be if the perturbation is small, then the spectral density of the emitted energy can be computed following a Taylor expansion, and the total radiated energy is then derived by integration over all modes. Again, the result was that there was a critical velocity $v_{\mathrm{thr}}=\left(1+k^{2}\right)^{-1 / 2}$ such that kinks traveling with velocities $v<v_{\text {thr }}$ did not emit any radiation at all, whereas in the opposite case the amount of emitted radiation decreased as $v \rightarrow 1$, diverging when $v=v_{\mathrm{thr}}$. Always within the framework of ISPT, Malomed and Tribelsky [10] were also able to show that dissipation could play a regularizing role, suppressing the divergence. As the results in Ref. [8] agreed with those in Refs. $[9,10]$, the existence of this threshold for radiation with its associated divergence was accepted, and the question of kink propagation on periodic potentials was 
regarded as basically solved. As mentioned above, it has to be borne in mind that the main issue of those early researches was to establish the proper foundations for a perturbative theory for solitons. Hence the question of the physical meaning and origin of the divergence was not addressed. Another unexplained point arises already from ISPT, which allows computation of the radiation nature. When this is done in our case, the radiation wave numbers turn out to be related to the perturbation one by a complicated equation (see, e.g., [2]), which, in particular, implies that radiation is emitted with a nonintuitive wave number $k^{-1}$ at the divergence. This prediction is difficult to understand physically. Let us recall at this point that a particlelike picture of kink propagation had been developed and had been largely successful so far [12] when compared to numerical experiments. If ISPT predictions for the radiation wave numbers were true, the reason for them must come from the wave nature of kinks. Consequently, the particle picture should be regarded as a major simplification and valid only in limited cases.

\section{KINK PROPAGATION ON PERIODIC MEDIA}

\section{A. Numerical results}

With the above scenario (and the question it poses) in mind, we carried out a number of numerical simulations looking in the first place for the proposed threshold. All the simulations we will be reporting on throughout the paper have been carried out taking periodic boundary conditions. The integration was performed with two different procedures, an adaptation of the energy conserving Strauss-Vázquez finite-difference scheme [13] and a fifth order, adaptive step size, Runge-Kutta integration [14] of the discretization of the PDE. The results were independent of the procedure, which is a satisfactory checking. We performed a careful search, paying attention to the fact that the predicted value was a first order calculation and that it may not be quantitatively accurate. On the other hand, the finite width of the simulated system may also be of relevance at this point, as its radiation spectrum structure is not identical to the continuum, infinite system [in particular, the lowest frequency in the model is restricted to be $\omega_{\min }^{2}=1+(2 \pi / L)^{2}, L$ being the length of the system]. Hence we monitored the amount of radiation emitted by the kink by making simulations with many different initial conditions, sweeping a range of initial velocities; if there was a threshold somewhere, there should be a change in the radiating power of the kink as it moved through it. The result was negative: No evidence for a threshold was found, even when the search was performed for a large range of initial velocities with a resolution of $10^{-2}$ for some choices of $k$. Examples of the outcome of the simulations are shown in Fig. 1 for three values of the potential wavelength: (a) of the order and (b) and (c) smaller than the kink width ( $\sim 6$ in our dimensionless units) at $v=v_{\mathrm{thr}}$. It has to be stressed that the predicted divergence does not depend on the strength of the perturbing potential $\epsilon$, but we also tried to make
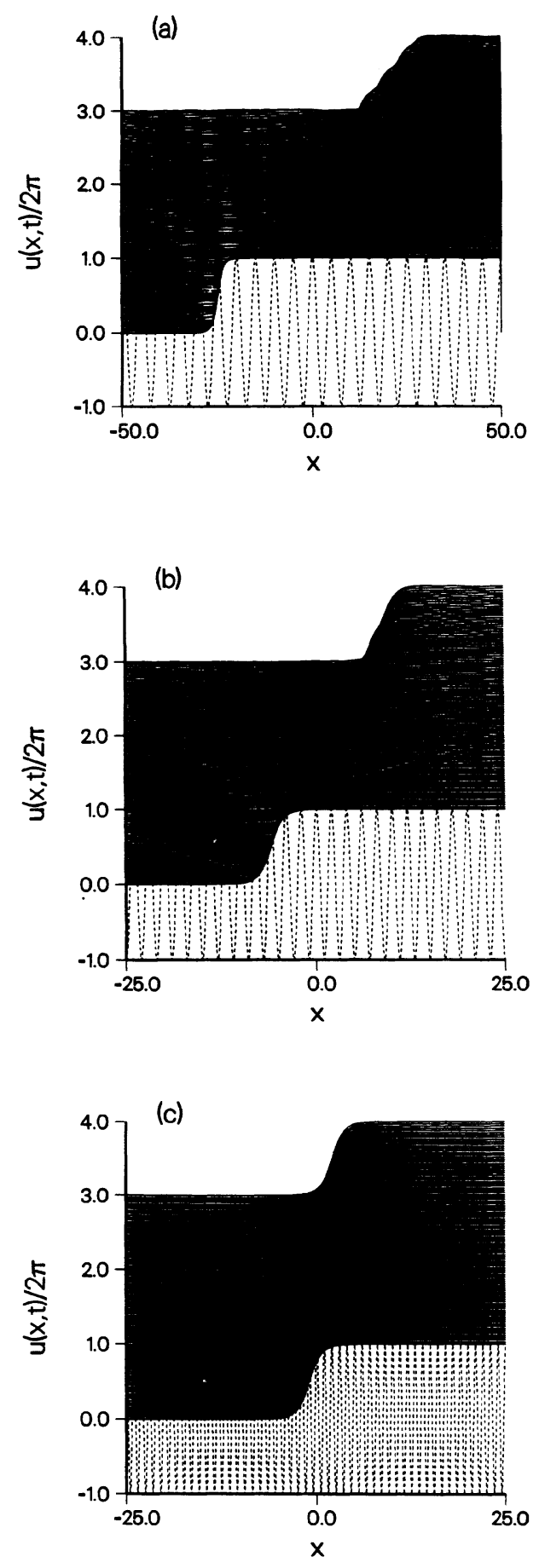

FIG. 1. Absence of radiative divergence for kinks propagating in the spatially periodic SG model. Parameters are (a) $k=2 \pi / 5$, initial velocity $v=v_{\mathrm{thr}}=0.387726 \ldots ;$ (b) $k=\pi$, initial velocity $v=v_{\mathrm{thr}}=0.091999 \ldots ;$ (c) $k=2 \pi$, initial velocity $v=v_{\text {thr }}=0.024704 \ldots$ [corresponding wavelengths are (a) 5 , (b) 2 , and (c) 1]. In all three cases, $\epsilon=0.4$. The amplitude of the emitted radiation is very small; due to the periodic boundary conditions, it can be seen reentering the simulation interval without any appreciable interaction with the kink. Only half of the simulation interval is shown in plots (b) and (c) to enlarge details. Time increases upwards with final time $t=100$. The potential is indicated by the dashed line (amplitude not to scale). 


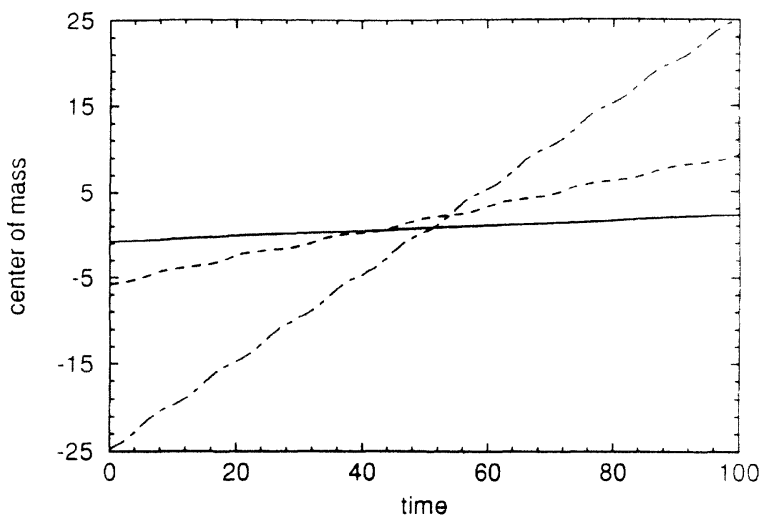

FIG. 2. Instantaneous center of mass positions as obtained from the simulations in Fig. 1. Dot-dashed line, $k=2 \pi / 5$; dashed line, $k=\pi$; solid line, $k=2 \pi$.

the effect more visible by increasing this parameter. Indeed, in Fig. 1, $\epsilon=0.4$, a value that is not very small, and the kinks seem unaffected except for a small amount of radiation and an oscillatory motion superimposed on its trajectory, which is shown in Fig. 2. It is interesting to note that the kink traveling on the short wavelength potential (c) appears not to be affected at all. This will be understood by means of the collective coordinate approach in Sec. IV. On increasing $\epsilon$ further, trapping behavior takes place, i.e., kinks are trapped by the potential and cannot propagate, but there is no strong emission of radiation [for an example, see Fig. 4(b), which will be discussed later]. Actually, this trapping can be of two very different kinds, as we will discuss in Secs. IV and V. Another interesting remark is that we also observed that kinks always emit radiation, even when moving at a very low velocity, far below the predicted threshold. A similar result arises from the work of Peyrard and Kruskal on highly discrete SG systems [15], where kinks propagate on the periodic potential coming from the PeierlsNabarro barrier, although this comparison should not be taken too literally as there are some differences between both problems, such as the existence of a maximum allowed frequency in the discrete one, for instance. It thus becomes evident that the features of kink propagation on periodic potentials are qualitatively different from the above perturbative analytical results. Interestingly, numerical simulations on a similar perturbation of the $\phi^{4}$ problem [16] seem to confirm the absence of this divergence. We will elaborate more on this when presenting our conclusions in Sec. VI.

\section{B. Theory}

In order to gain insight into the numerical observations, we developed a perturbative approach for this problem, following a similar approach to that given by Fogel et al. [12]. To this end, we perform a Lorentz transformation and rewrite (1) in the rest frame of the soliton (i.e., the reference frame moving with the speed of the unperturbed soliton $v$ )

$$
u_{t t}-u_{x x}+\{1+\epsilon \cos [k \gamma(x+v t)]\} \sin u=0,
$$

with $\gamma \equiv\left(1-v^{2}\right)^{-1 / 2}$ the Lorentz factor. Here we consider $\epsilon \ll 1$, so the perturbative term may be treated by assuming a solution of the form

$$
u(x, t)=u_{v}(x)+\epsilon u^{(1)}(x, t),
$$

where $u_{v}(x) \equiv 4 \tan ^{-1}\left(e^{x}\right)$ is the unperturbed SG kink. For completeness, we now recall how the most appropriate basis in which to expand $\epsilon u^{(1)}(x, t)$ is obtained. Introducing the Ansatz (3) in Eq. (2) without the perturbation term, linearizing in the small quantity $u^{(1)}(x, t)$, and separating time and space by introducing $u^{(1)}(x, t)=$ $f(x) e^{-i \omega t}$, we are left with the following eigenvalue problem for $f(x)$ :

$$
\left[-\frac{d^{2}}{d x^{2}}+\left(1-2 \operatorname{sech}^{2} x\right)\right] f(x)=\omega^{2} f(x) .
$$

This is a well known eigenvalue problem [17]; there exists exactly one bound state, with $\omega_{b}=0$, and a continuum of scattering states with $\omega_{\kappa}^{2}=1+\kappa^{2}$; the corresponding normalized eigenfunctions are

$$
\begin{aligned}
f_{b}(x) & =2 \operatorname{sech} x, \\
f(\kappa, x) & =\frac{1}{\omega_{\kappa} \sqrt{2 \pi}} e^{i \kappa x}(\kappa+i \tanh x) .
\end{aligned}
$$

These eigenfunctions have a very clear physical meaning. The bound state $f_{b}(x)$ is associated with the Goldstone translation mode of the soliton, whereas the continuum eigenfunctions $f(\kappa, x)$ are the radiation modes (see [12] for a detailed discussion). Besides, these functions form an orthogonal basis since the corresponding operator is self-adjoint. We will make use of this fact to deal with our problem. In terms of this basis, the first order correction can be split into two parts, namely,

$$
u^{(1)}(x, t)=u^{(\text {trans })}(x, t)+u^{(\mathrm{rad})}(x, t),
$$

where

$$
\begin{aligned}
u^{(\text {trans })}(x, t) & =\frac{1}{8} \phi_{b}(t) f_{b}(x), \\
u^{(\mathrm{rad})}(x, t) & =\int_{-\infty}^{\infty} d \kappa \phi(\kappa, t) f(\kappa, x) .
\end{aligned}
$$

To find the amplitudes $\phi_{b}(t)$ and $\phi(\kappa, t)$, one again introduces the Ansatz (3) in Eq. (2), linearizes, and Fourier transforms in time; subsequent projection yields

$$
\begin{aligned}
& \ddot{\phi}_{b}(t)= 4 \int_{-\infty}^{\infty} d x \cos [k \gamma(x+v t)] \frac{\sinh x}{\cosh ^{3} x}, \\
& \ddot{\phi}(\kappa, t)+\left(1+\kappa^{2}\right) \phi(\kappa, t) \\
&=2 \int_{-\infty}^{\infty} d x \cos [k \gamma(x+v t)] \\
& \times \frac{e^{-i \kappa x}(\kappa-i \tanh x)}{\sqrt{2 \pi\left(1+\kappa^{2}\right)}} \frac{\sinh x}{\cosh ^{2} x} .
\end{aligned}
$$

It now remains to solve Eqs. (8) and invert the various 
transforms needed to arrive at them. In the following, we discuss translation and radiative parts in (6) separately. Let us start with the simplest one, i.e., the translation mode contribution. Note that (8a) is, after performing the integration, nothing but the Newton's law for a timedependent force. Its solution may be readily found, and finally one obtains

$$
u^{(\text {trans })}(x, t)=\frac{\pi}{2 v^{2} \sinh (k \gamma \pi / 2)} \sin (k \gamma v t) \operatorname{sech} x .
$$

Recalling that we are working in the unperturbed soliton reference frame, this is a localized oscillatory motion superinuposed on its otherwise constant trajectory. Now, let us remark that the prefactor inıplies that short wave- length $(k \rightarrow \infty)$ perturbations will have no effect on the motion of the center of the soliton, which is also in good agreement with our simulations in Fig. 2. This behavior can be understood in terms of a "smoothing" of the potential: The kink, having a width much larger than the perturbation wavelength, experiences only an effective averaged force, whose aniplitude vanishes exponentially for large $k$ (see Sec. IV; see also related comments in $[4,6])$.

Equation $(8 \mathrm{~b})$ for the $\kappa$-mode radiative contribution can also be solved. After computing the integral in the right hand side of Eq. (8b), one is left with the Newton's law for a forced harmonic oscillator. This allows the determination of $\phi(\kappa, t)$ and substitution of it in Eq. (7b) to find the total radiative contribution:

$$
u^{(\mathrm{rad})}(x, t) \equiv \frac{1}{4}\left[\tanh x-\frac{\partial}{\partial x}\right] \int_{-\infty}^{\infty} d \kappa \frac{1+\kappa^{2}-k^{2} \gamma^{2}}{\left(1+\kappa^{2}\right)\left(1+\kappa^{2}-k^{2} \gamma^{2} v^{2}\right)}\left[\frac{e^{i k \gamma v t}}{\cosh [\pi(k \gamma-\kappa) / 2]}+\frac{e^{-i k \gamma v t}}{\cosh [\pi(k \gamma+\kappa) / 2]}\right] e^{i \kappa x}
$$

It is possible to deal with the integral in (10) in the complex plane: When $x>0$ in the upper half plane and when $x<0$ in the lower half plane. The pole structure of the integrand will completely determine the total radiative contribution. In particular, we will see that radiation only appears for some special values of the system parameters.

We take $x>0$ in what follows (the opposite case is treated in the same way). Accordingly, the integral has to be analyzed in the upper half complex plane. The pole structure of the integrand is depicted in Fig. 3. All poles are simple and their locations are $z_{0} \equiv+i, z_{1} \equiv$ $i \alpha \equiv+i \sqrt{1-k^{2} \gamma^{2} v^{2}}$, and $z_{n}^{ \pm} \equiv \pm k \gamma+i(2 n+1), n$ being a non-negative integer. For the sake of clarity we treat each pole separately.

(i) The first pole $z_{0}=+i$ is constant and does not change when the system parameters change. Since this pole is purely imaginary, it is imnediately seen that the contribution of the residue at $z_{0}$ is exponentially localized around the kink center. This term does not give rise to any radiation but rather to time-dependent corrections of the kink shape.

(ii) The family of poles $z_{n}^{ \pm}$depends on the perturbation wave number $k$ and on the kink velocity through the Lorentz factor $\gamma$. However, they always have a positive imaginary part, thus leading again to exponentially localized contributions. Therefore, the $z_{n}^{ \pm}$poles also do not produce any true radiative correction.

(iii) The remaining pole is the key one. If $\alpha^{2} \equiv$ $1-k^{2} \gamma^{2} v^{2}>0$, the same reasoning applied to the other poles holds, and there is no radiation. It is worth mentioning that localized oscillations around the kink center, predicted from the contributions of $z_{0}, z_{1}$ ( $\alpha$ real), and $z_{n}^{ \pm}$, were already evident in our numerical simulations, as shown in Fig. 1. For fixed $k$, as $v$ increases, the pole moves down the imaginary axis, and at the critical value $v_{\mathrm{thr}} \equiv\left(1+k^{2}\right)^{-1 / 2}$ it lies at the origin of the complex plane. For kink velocities $v>v_{\text {thr }}$ the pole is purely real and then it does give rise to a radiative contribution, whose form is given by (with $\beta \equiv \sqrt{k^{2} \gamma^{2} v^{2}-1}$ a real number)

$$
\begin{aligned}
u_{\beta}^{(\mathrm{rad})}= & \frac{\pi}{4 \gamma^{2} v^{2}}\left(1-\frac{i}{\beta} \tanh x\right) \\
& \times\left[\frac{e^{i(k \gamma v t+\beta x)}}{\cosh [\pi(k \gamma-\beta) / 2]}+\frac{e^{-i(k \gamma v t-\beta x)}}{\cosh [\pi(k \gamma+\beta) / 2]}\right] .
\end{aligned}
$$

This expression tells us that radiation occurs whenever $\beta$ is real $\left(v>v_{\text {thr }}\right)$, and this radiation is the superposition of two linear waves of different amplitudes, traveling in opposite directions but with the same phase velocity.

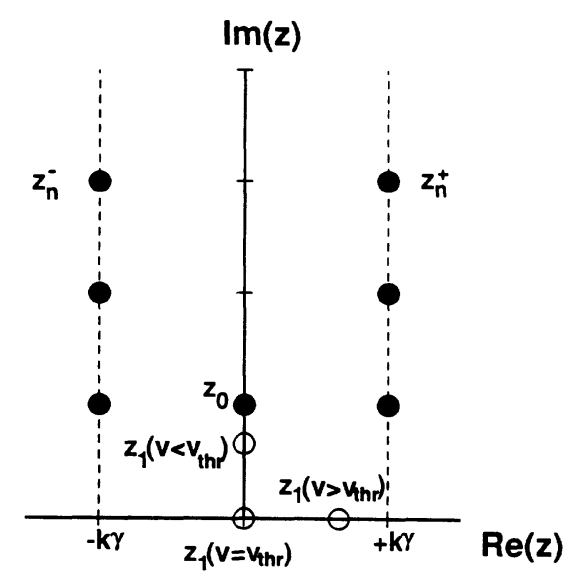

FIG. 3. The pole structure of the radiation contribution. Filled circles mark the location of the poles which give rise to corrections localized around the soliton. Empty circles denote the locations of pole $z_{1}$ as the velocity changes. Only when $z_{1}$ becomes real $\left(v>v_{\text {thr }}\right)$ does it originate propagating wavelike corrections. See text for further explanation. 


\section{Discussion}

To this point, it appears that our perturbative calculation leads exactly to the same prediction as those in $[8,10]$, namely, that there is a critical velocity $v_{\mathrm{thr}} \equiv$ $\left(1+k^{2}\right)^{-1 / 2}$ below which kinks do not radiate and above which they do. At that precise velocity, the amplitude of the emitted radiation diverges; notice that $\beta$ vanishes as $v$ approaches $v_{\text {thr }}$ from above and consequently the prefactor in Eq. (11) goes to infinity. However, this apparent equivalence is not so. The crucial difference arises when one looks more carefully at Eq. (11): As $v_{\text {thr }}$ is approached, not only the amplitude of the emitted wave diverges, but also its wavelength $2 \pi / \beta$. Then, we are faced with something similar to an "infrared" divergence, and usually those do not have a real physical meaning. We will show immediately that this is indeed the case here, but let us first comment on the reasons why our calculation provides us with this physically relevant result that was not transparent in the previous approaches. As for the GFPT computation [8], they compute the first order correction to the field much as we do here (actually the two approaches are basically the same in the beginning), but they do not use the natural translation mode-radiation basis, so they cannot separate the different contributions and are therefore led to an expression they cannot analyze in detail; as we already pointed out, they merely remark that their calculations are invalid in the vicinity of the divergence, as they assumed the correction should be small. On the other hand, ISPT $[9,10]$ yields a different result than ours in spite of using a suitable basis because the integration over $\kappa$ is made in an incoherent fashion, i.e., integrating over emitted energy instead of emitted amplitude (we notice in passing that many ISPT results are obtained by this same means). When the integration over radiation modes is made coherently as shown here, the result changes due to the superposition of different modes. These reasons lead us to believe that, although admittedly the early perturbative work was mathematically sound, the calculation we present here is the physically correct first order result.

Now that we have a reliable perturbative calculation, we need to understand what is the nature of the divergence. To make progress, it is very important to turn to the form of our starting Eq. (1) with dimensions, namely,

$$
u_{t t}-c_{0}^{2} u_{x x}+\omega_{0}^{2}[1+\epsilon \cos (k x)] \sin u=0,
$$

where $c_{0}$ and $\omega_{0}$ are a velocity and a frequency characteristic of the particular physical context. Redoing the calculations with dimensions transforms the divergence condition $k \gamma v_{\mathrm{thr}}=1$ into $k \gamma_{0} v_{\mathrm{thr}}=\omega_{0}\left[\gamma_{0}=\right.$ $\left.\left(1-v^{2} / c_{0}^{2}\right)^{-1 / 2}\right]$. This immediately clarifies what happens: The divergence occurs when the velocity of the kink is such that the time it takes to travel through a wavelength of the potential, $T_{0}=\lambda /\left(\gamma_{0} v_{\mathrm{thr}}\right), \lambda=2 \pi / k$, is exactly the period of the lowest frequency phonon $T_{0}=2 \pi / \omega_{0}$. If the velocity is lower than $v_{\mathrm{thr}}$, the kink will not be able to excite phonons, whereas when its velocity is higher, it can and will subsequently radiate. At $v_{\text {thr }}$, the excited radiation is that of the lowest phonon and it has infinite wavelength and velocity, as predicted by our calculation. This natural picture of kinks exciting radiation according to the frequency of their propagation through a potential wavelength becomes therefore the likely candidate to explain the divergence. On the other hand, now it also becomes clear the divergence of the energy at $v_{\mathrm{thr}}$ : It diverges because of the infinite contribution arising from the infinite wavelength mode when integrated over the whole $x$ axis. This agrees with GFPT and ISPT results whose only difficulty was not to specifically identify the mode responsible for the divergence.

In spite of this clarification, the most significant question is not answered yet: Why do numerical simulations disagree with this calculation, which seems to allow for a simple and physically reasonable interpretation? By looking again at Figs. 1 and 2, it is easy to realize that the flaw of the perturbative calculation is at its very root: We are computing first order corrections around a kink moving at a constant velocity $v$, and this condition never holds. Whatever the starting position of the kink is, it will behave like a particle in the sense that it will be accelerated or decelerated depending on whether it travels towards a minimum or a maximum of the potential. In fact, the translation mode correction itself is describing this: The kink velocity, in its reference frame, is not zero but rather it oscillates between positive and negative values. It is not a surprise, then, that first the resonance condition we have obtained is never matched, and second that the kink emits radiation at any velocity, because it is accelerating or decelerating. Of course, we should note that this is a perturbative calculation including only first order terms; the possibility still remains that the divergence is suppressed by higher order nonlinearities.

\section{COLLECTIVE COORDINATE APPROACH}

The above numerical results and the subsequent perturbative calculation strongly suggest that SG kinks behave as pointlike particles in the presence of a periodic parametric potential like the one we deal with here. Therefore, it is natural to try to describe those results by means of the collective coordinate formalism. This approach was first proposed in [12] and it has been applied recently to SG breathers on periodic potentials [5] as well as to NLS [7] equations with the same perturbation. In both cases the analytical predictions turned out to be in very good quantitative agreement with numerical simulations: For instance, in Ref. [5] the threshold for breather breakup into a kink-antikink pair was predicted with an accuracy better than $0.1 \%$. On the other hand, the calculation in Ref. [7] predicted the appearance of the so called "soliton chaos," verified by simulations of the full PDE. In our present problem, the advantage we have is that, due to the simpler nature of the kink, we will be able to compute the effective potential not only for kinks at rest but also for moving kinks.

The basic idea of the collective coordinate formalism is very simple: To reduce a complicated problem with an infinite number of degrees of freedom, posed in terms of a PDE, to a much less complex problem with a few de- 
grees of freedom [and correspondingly described in terms of ordinary differential equations (ODE's)]. There are a number of ways to do this, and different quantities can be chosen to play the role of collective coordinates describing the motion of the nonlinear excitation as a whole. For our problem, it is enough to simply consider the kink center as our collective coordinate for the kink. Its motion will be then governed by an effective potential that can be computed by integrating the perturbative contribution to the Hamiltonian over the kink profile, i.e.,

$$
V_{\text {eff }}\left(x_{0}, t\right)=\epsilon \int_{-\infty}^{\infty} d x\left[1-\cos u_{v}\left(x-x_{0}, t\right)\right] \cos k x,
$$

where $u_{v}\left(x-x_{0}, t\right)$ denotes now a kink moving with constant velocity $v$ and centered at $x_{0}$. This integral can be easily evaluated and yields

$$
V_{\text {eff }}\left(x_{0}, t\right)=2 \epsilon \frac{k \pi}{\gamma^{2} \sinh (k \pi / 2 \gamma)} \cos \left[k\left(x_{0}+v t\right)\right] .
$$

From Eq. (14) we see that the potential experienced by the particle equivalent to the kink is basically the same perturbation potential that appears in the PDE (1), although the prefactor in front of it is quite complicated. The simplest dependence of this prefactor is on the wave number. It can be immediately seen that when $k \rightarrow 0$ (long wavelength limit) the effective potential prefactor reduces to $4 \epsilon / \gamma$ and subsequently $V_{\text {eff }}$ becomes closer to the perturbative one; in the opposite limit, $k \rightarrow \infty$, the sinh term makes the effective potential vanish exponentially. This is in agreement with what we have learned so far: Looking at Fig. 1, it can be seen that the short wavelength potential has no effect on the kink (c), whereas the motion on long wavelength perturbations resemble that of a particle on the bare potential. To phrase in the terminology introduced in Ref. [4], the behavior of the kink in these cases is that of a "bare" (long wavelength) or a "renormalized" (short wavelength) particle. It is also important to notice that this result agrees with the perturbative calculation we described in Sec. III B [see Eq. (9)], as it was to be expected. There we showed that the correction to the center of mass motion was basically an oscillatory term, implying that the velocity of the center of mass oscillates around some mean value. This is precisely the same kind of trajectory followed by a pointlike particle in the potential in Eq. (14) (at least if the velocity is not too close to 1 ).

Nevertheless, it is worth pursuing this agreement a bit further, by studying the threshold for kinks to propagate in this kind of potential. The easiest way to compute the threshold is by equating the kinetic energy of the kink to the maximum of the effective potential, provided we restrict ourselves to the nonrelativistic limit $\left(v^{2}\right.$ not too close to 1) to keep the kink mass constant. This will give us the maximum potential height over which a kink that starts from a point at which the perturbation is zero with a certain velocity is able to overcome the nearest top point. Using the fact that the mass of a not too fast kink is 8 in our units, we find that the threshold is given by

$$
\epsilon_{\mathrm{thr}}=\frac{2 v^{2} \gamma^{2}}{k \pi} \sinh \frac{k \pi}{2 \gamma}
$$

In the same way, we could have computed the threshold velocity for a given strength of the potential, but we prefer to check our predictions this way because the presence of $\gamma$ makes the other possibility more complicated. We compared this prediction to numerical simulations. We show an example of this comparison in Fig. 4, where
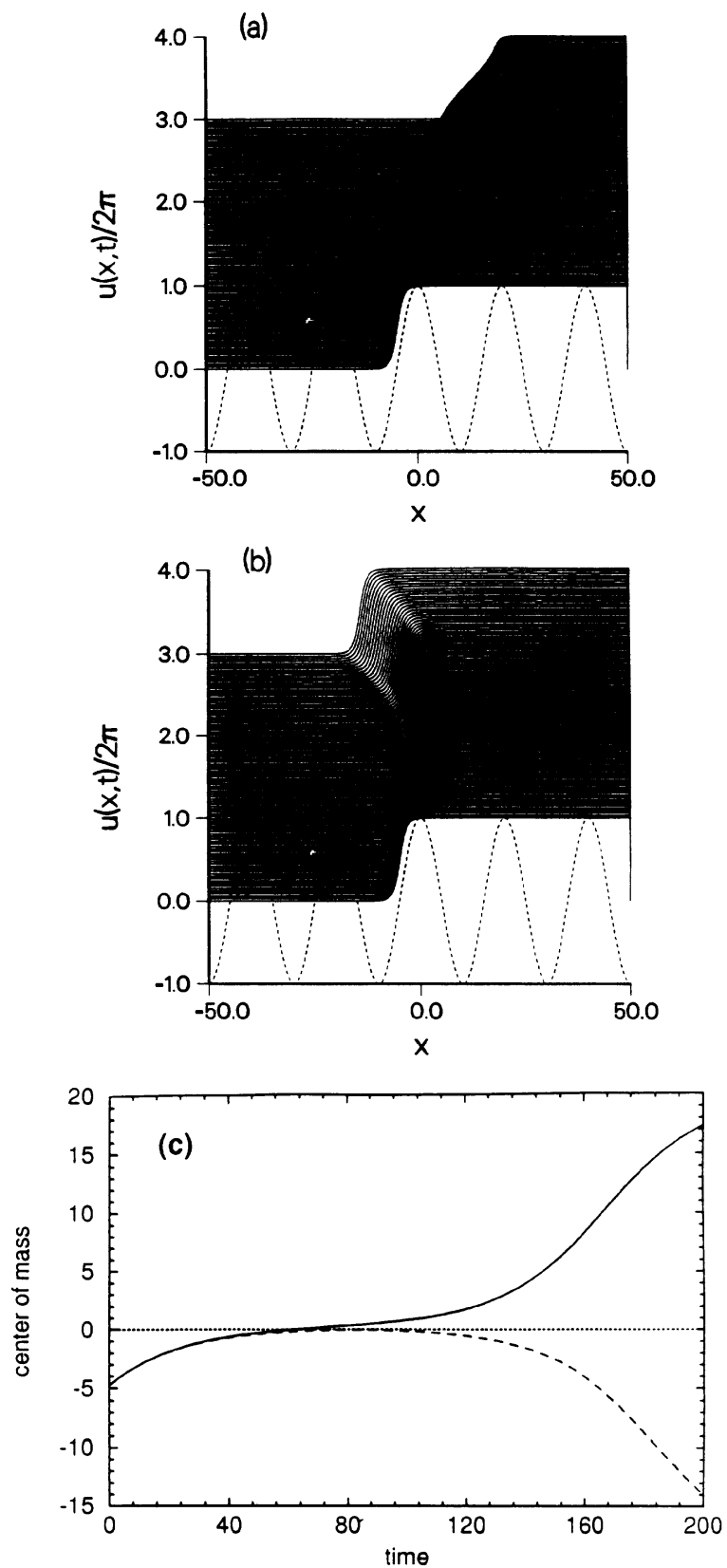

FIG. 4. An example of the verification of the collective coordinate approach predictions. For a kink starting from a midpoint of a potential of wavelength 20 , with velocity 0.2 , the predicted threshold for propagation is $\epsilon_{\mathrm{thr}}=0.0424$. (a) $\epsilon=0.43$ and the kink propagates; (b) $\epsilon=0.435$ and the kink is reflected by the potential maximum; (c) center of mass motion for better comparison of both cases; solid line corresponds to the simulation in (a) and dashed line to that in (b). Final time is $t=200$. Notice the absolute absence of radiation in this phenomenon. 
we study the propagation of a kink with initial velocity 0.2 (i.e., in a nonrelativistic situation) on a potential of a moderately long wavelength. The predicted threshold for kink propagation as given by Eq. (15) is $\epsilon_{\mathrm{thr}}=0.0424$; from Fig. 4 we see that the numerical result is bounded by $0.043<\epsilon_{\mathrm{thr}}<0.0435$, meaning that the error in our prediction is at most of $2 \%$, which is quite satisfactory. We have checked several other cases, which we summarize in Fig. 5; excellent agreement is always found, even for very large values of the perturbation potential.

To conclude this section on the collective coordinate treatment of the problem, we discuss another prediction of it that it is numerically verified, relativistic effects playing now the relevant part. By looking again at Eq. (14), it can be realized that the presence of $\gamma$ in the potential may give rise to singularities in the neighborhood of the maximum velocity $v=1$. To check whether this is so, we integrated numerically the ODE obtained from applying second Newton's law to $V_{\text {eff }}$ and found that if the initial conditions were those of a particle starting at the top of a large potential, the velocity of that particle would grow as it slides down the potential; of course, if the potential is large enough, the velocity can reach $v=1$ : In those cases the numerical integration of the ODE broke down. The question then arises whether this is an artifact of our collective coordinate approach or there is a related phenomenon in the full PDE. We actually found that this ODE prediction is verified, as we show in Fig. 6. In this simulation, the initial condition was a kink at a maximum of the potential with initial velocity 0.1 so as to start the motion from the stable point. As it moves to the nearest well, it accelerates and, eventually, its velocity becomes very close to 1 , implying that the kink cannot accelerate further. Then it is trapped at an intermediate point of the potential instead of continuing its motion to the bottom. The existence of this counterintuitive phenomenon shows in a very dramatic way the value of a simple approach such as the collective coordi-

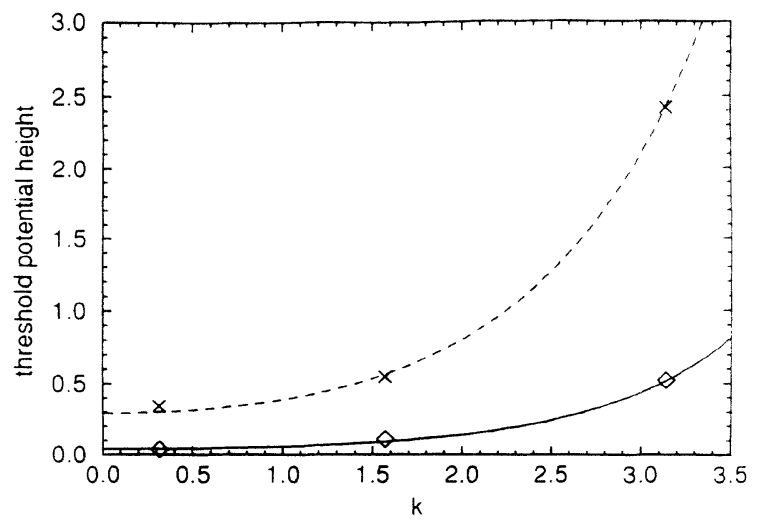

FIG. 5. Comparison of the effective potential prediction with numerical simulations for a number of cases. Solid line, threshold for initial velocity 0.2 as a function of the potential wave number; dashed line, the same for initial velocity 0.5 ; diamonds and crosses, points obtained from the numerical study; error bars in the numerical threshold are of the order of the size of the symbols.
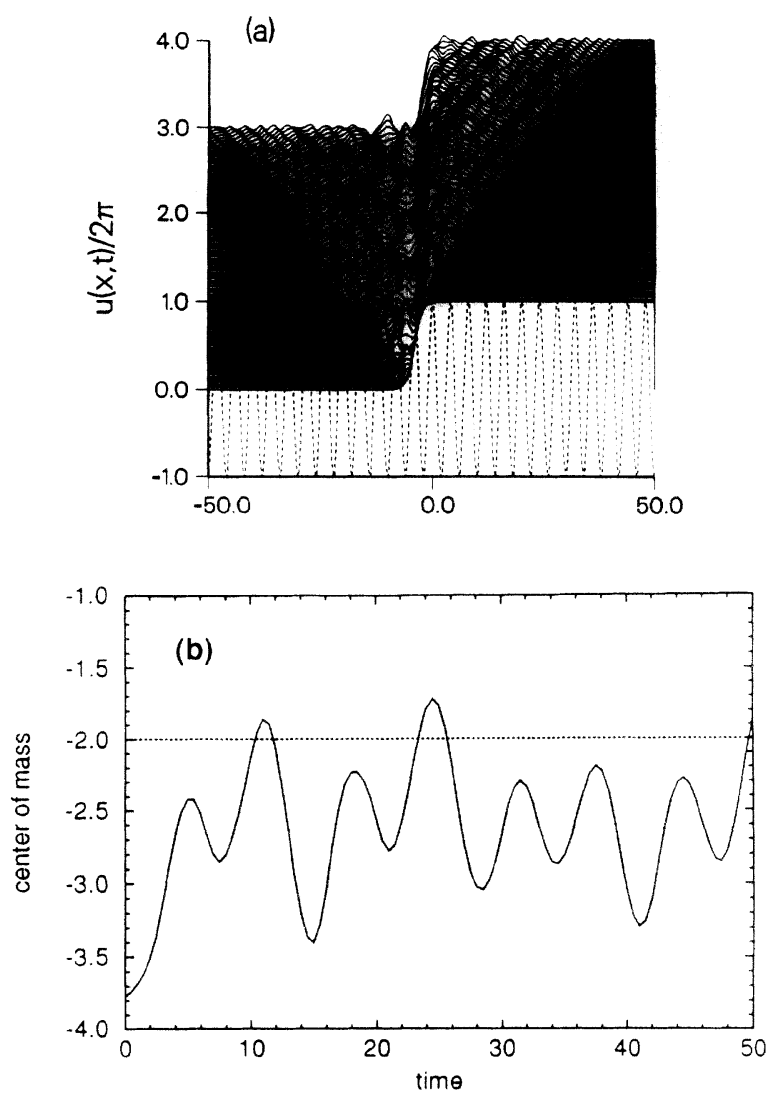

FIG. 6. Trapping of a kink at an intermediate position in the potential instead of at the bottom. $\epsilon=2$ and the kink starts with velocity 0.1 . The amount of emitted radiation is large due to the fast acceleration of the kink in this large perturbation. (a) Time evolution of the kink; (b) time evolution of the center of mass. The neighboring minimum is at $x=-2.0$ (indicated by the dashed line) and the kink oscillates around approximately $x=-2.5$. The final time is $t=50$.

nate formalism to help understand complicated nonlinear phenomena.

\section{LENGTH-SCALE COMPETITION}

\section{A. Numerical experiments}

The numerical and theoretical analysis described so far allowed us to achieve a quite good understanding of the periodically perturbed SG problem, at least of the basic phenomenology. With that background in mind, we then turned to the initial motivation for this work, namely, to study kink propagation in periodic potential as a step towards the much more complicated problem of breather propagation on periodic media [4]. In principle, we did not expect length-scale competition to arise in this problem, as kinks are very robust objects (which is further confirmed by our above results, in particular by the success of the collective coordinate approach). On the other hand, opposite to the case of the breather, kink widths 
do not vary much when changing the only parameter governing it, the kink velocity. Of course, when approaching the maximum velocity, Lorentz contraction of the kinks will make them vanishingly small, but that is a regime in which is very difficult and time consuming to carry out good numerical simulations, so we did not address the problem in that limit. Therefore, the kinks we are usually dealing with have a width of about 6 in our dimensionless units. Our purpose was to perform some simulations in the high perturbation regime to see whether any light could be shed on the related breather problem.

The numerical experiments we made were as follows. We studied several potentials of different wavelengths, ranging from 0.5 to 20 , i.e., from much smaller than the kink width to roughly three times its width. The initial condition was always a kink at one top of the potential; different velocities were considered. A summary of these experiments in the most interesting range of potential wavelengths is shown in Figs. 7 and 8 . Figure 7 shows the motion of the center of mass of the kink on different potentials. The motion in the small or large wavelength limits has been already discussed and it is again seen here. However, a more interesting phenomenon is also evident, namely, kink trapping [or even reflection in the case of wavelength 3 ; see Fig. 8(b)]; this trapping was

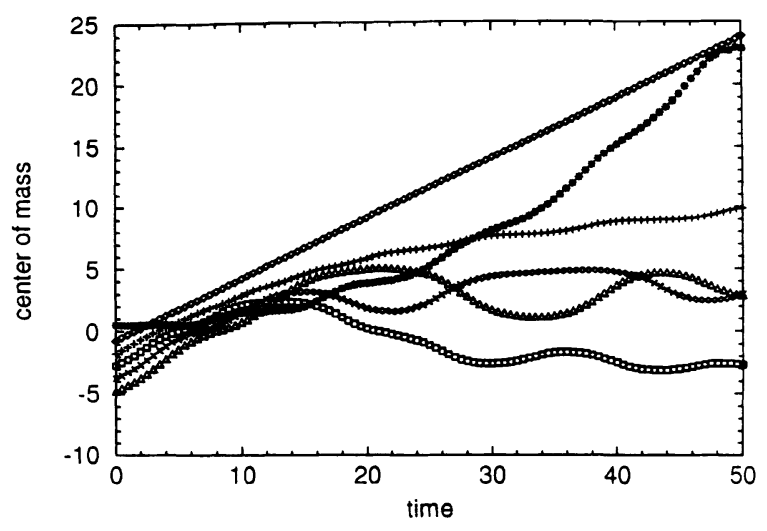

FIG. 7. Length-scale competition. Center of mass evolution of a kink on potentials of different wavelengths, always with $\epsilon=0.7$ and initial velocity 0.5 . Wavelengths are $\diamond, 1$; ,$+ 2 ; \square, 3 ; \times, 4 ; \triangle, 5$; and $*, 6$. See text for explanation.

not to be expected because kinks start from a maximum of the potential and with a large initial velocity (in Fig. 7 it was 0.5). Regarding this point, we have to stress here that this trapping is of a different kind than the one discussed in the preceding section, which was clearly a nonradiative process. Besides, the trapping depends cru- (a)

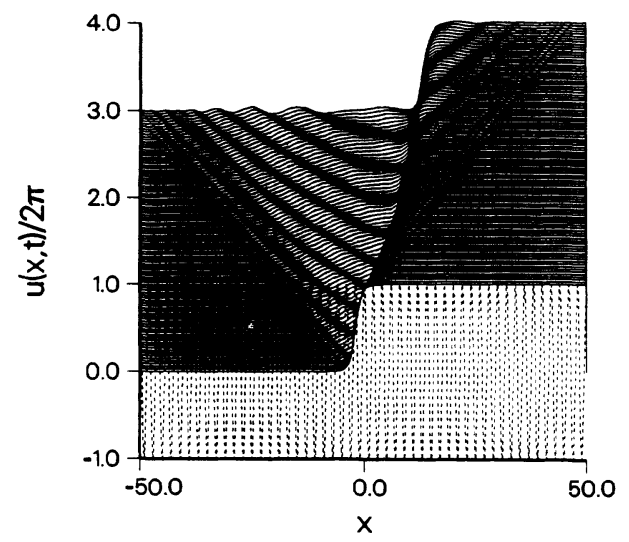

(c)

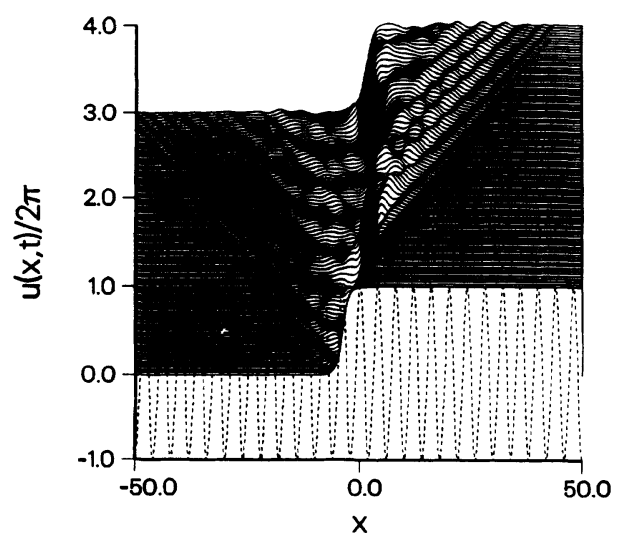

(b)

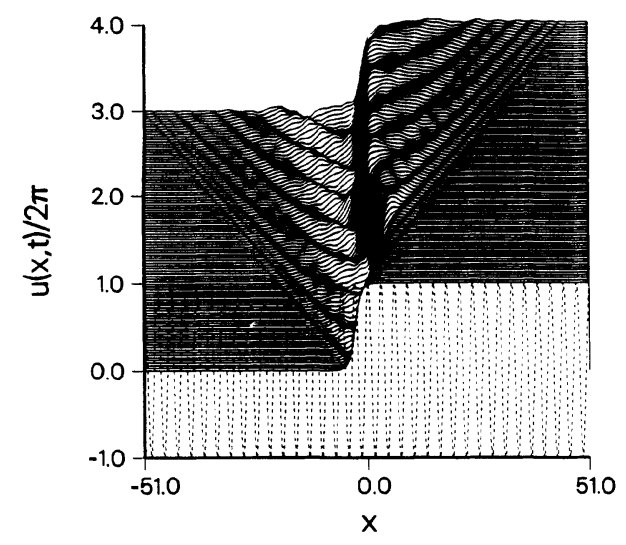

(d)

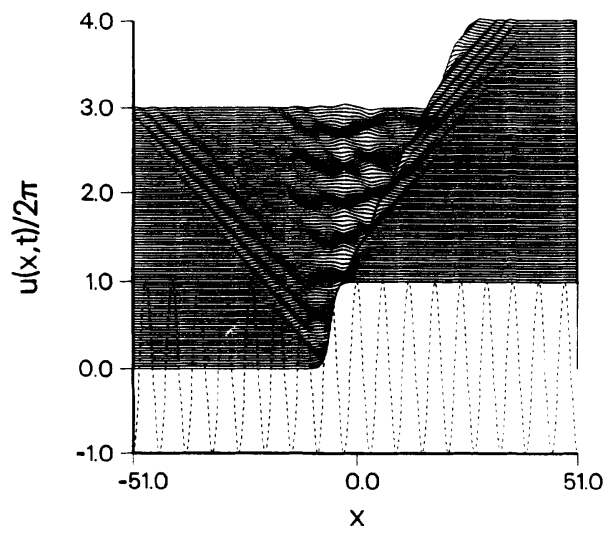

FIG. 8. Time evolution for some of the kinks in Fig. 7. Wavelengths are (a) 2; (b) 3; (c) 4; and (d) 6. Notice the different kind of radiation emitted in each case. Notice also that the kink in (b) goes back over one maximum of the potential probably due to resonant interaction with radiation left behind. The final time is $t=50$. 
cially on the wavelength of the potential. Thus, for instance, in the case of wavelength 2 [Fig. 8(a)], the kink is able to propagate over six wells, whereas when the wavelength was 4 or 5 [Figs. $8(\mathrm{c})$ and $8(\mathrm{~d})$ ], it was trapped already on the second well. This dependence clearly indicates that length-scale competition is to same extent present also in the kink problem. This hint is further supported by our previous work for the breather case [4], which showed that this competition was most effective when the wavelength of the perturbation was around half the breather width or slightly larger. This is also the case in these simulations. Another common feature between both problems is that the outcome of a simulation depends very sensitively on the initial condition. This can be understood from the reflection case in Fig. 8(b): For the kink to jump back over one potential wavelength it is necessary that it meets the radiation it left behind in the appropriate phase to gain energy from it, and this evidently depends crucially on the initial velocity, as we checked in our simulations. Hence we conclude that these phenomena are a manifestation of length-scale competition.

\section{B. Linear stability analysis and discussion}

The numerical findings we have described in the preceding subsection are of great importance: The existence of processes governed by length-scale competition in the SG kink case opens the possibility of a deeper study of the mechanisms through which this competition affects the kink evolution. The relevance of this comes from the fact that, when studying the SG breather problem [4], we were not able to carry out this deeper analysis due to the more complicated nature of the breathers, namely, their intrinsic internal dynamics which severely complicate linear stability analysis. But after showing that this competition also affects kinks, we can certainly study their linear stability analysis and, consequently, obtain an understanding of the mechanisms underlying length-scale competition.

We tested the stability of the analytical continuum solutions as well as of the numerical solutions in the following way. Let the solution to discretization of the SG equation (1) be $u_{i}=u_{i}^{(0)}+v_{i}$, where $u_{i}^{(0)}$ is either the discretized version of the continuum kink or the true minimum energy static solution of the perturbed SG equation (1) obtained numerically and $v_{i}$ is a small discrete-valued function whose time dependence is given by $\sin (\omega t)$; the index $i$ runs over the $N$ points of the discrete lattice. The discrete version of the perturbed problem (1) is given by

$$
\ddot{u}_{i}-a^{-2}\left(u_{i+1}-2 u_{i}+u_{i-1}\right)+[1+\epsilon \cos (k a i)] u_{i}=0,
$$

where $a$ is the lattice spacing. Substituting the proposed form for $u_{i}$ in Eq. (16) and linearizing we get

$$
\mathbf{\Omega v}=\omega^{2} \mathbf{v}
$$

where $\mathbf{v}$ is the vector containing the $v_{i}, i=1, \ldots, N$, and
$\boldsymbol{\Omega}$ is an $N \times N$ matrix given by

$$
\boldsymbol{\Omega}_{i j}=\left\{\begin{array}{l}
2+[1+\epsilon \cos (\text { kai })] \cos \left(u_{i}^{(0)}\right), \text { if } i=j \\
-1, \text { if } i=j \pm 1 \\
0, \text { otherwise. }
\end{array}\right.
$$

In this formulation, the modes for the linear excitations around the shape $u^{(0)}$ are obtained simply by solving for the eigenvalues $\omega^{2}$ of the matrix $\Omega$. We did this for all the wavelengths we were studying, taking for $u^{(0)}$ the exact continuum SG kink at the top or at the bottom of the potential as well as the numerically obtained solutions at similar positions.

The results for our numerical linear stability analysis are shown in Fig. 9 for some of the relevant wavelengths [18]. There are a number of interesting features which deserve comment. First, let us consider the spectra for the exact continuum shapes. When placed at the top of the potential, this gives rise to a negative lowest eigenvalue $\omega^{2}$, indicating that this continuum kink is not an exact solution of the discrete problem and has a tendency to relax to the correct one by emitting a burst of radiation. The shape at the bottom of the potential does not show this negative eigenvalue but instead a single bound state with frequency $\omega^{2} \approx 0.3$. This corresponds to a shape mode, similar to that present in unperturbed $\phi^{4}$ kinks, and it actually shows up in simulations: Initial data placed at a potential well (an exact continuum kink) exhibit a static center of mass but a general oscillating shape. This is easily understood if one realizes that in this range of potential wavelengths, different parts of the kink undergo the action of very different perturbation values (which can be even positive or negative contributions). In response to these gradients, the continuum kink oscillates. These isolated states characteristic of the continuum kinks disappear when we analyze static numerical solutions. It is seen from Fig. 9 that in that situation spectra are composed of bands. Actually, this is a common feature to all analyzed shapes, including the continuum ones, and it could be expected in view of the following argument: Far from the kink center, which only spans a small fraction of the lattice sites, the nonlinear contribution to the linearized discrete problem for $v_{i}$ vanishes, and one is left with what is a standard Floquet (Bloch) problem. The corresponding structure is very well known, and in fact it is very much like the ones we show here, with gaps at positions that depend on the potential wave number and gap amplitudes that depend on the potential strength $(\epsilon)$.

By comparing the spectral structure we have obtained to the outcome of the numerical simulations (Fig. 9) and especially to the radiation emission (Fig. 8), the mechanism for kink destabilization can be inferred. In the case of small wavelength potentials, the spectrum is very similar to the unperturbed SG one [see Fig. 9(a)]. Therefore, the behavior of the kink is very similar to the continuum one moving in a discrete lattice, the periodic potential then being nothing but the Peierls-Nabarro barrier, as we already mentioned. In that case the kink is known to radiate [15] and correspondingly decelerate until it is eventually trapped in a potential well [Fig. 8(b)]. When 

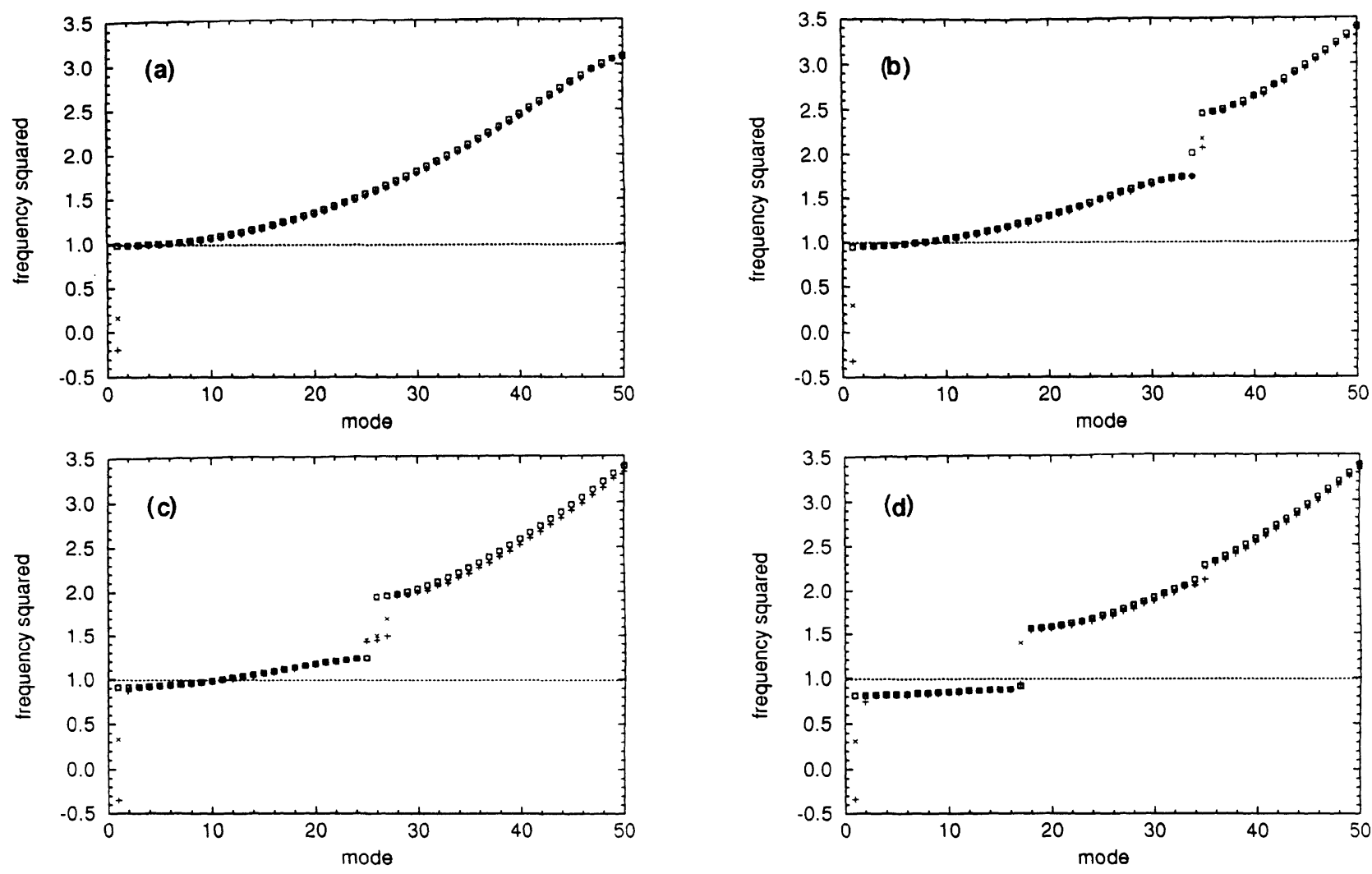

FIG. 9. Frequency spectra for kinks on periodic potentials. See text for details on how it is computed. Spectra for numerical shapes are plotted with $\diamond$ (potential maximum) and $\square$ (potential minimum). Spectra for analytical shapes are plotted with + (potential maximum) and $\times$ (potential minimum). Wavelengths are (a) 2, (b) 3, (c) 4, and (d) 6. Notice in this last case that the whole first branch of the spectrum is below the phonon band.

the wavelength is smaller, the height of the effective potential seen by the kink is so small [due to the sinh term in Eq. (14)] that this effect is hardly noticeable (hence the perfect constant motion of the kink on the smallest wavelength potential in Fig. 7). As the wavelength increases, more and more modes move below the phonon band, inducing shape oscillations of the kink, and as a consequence of this motion, long wavelength radiation is emitted [clearly seen in Figs. 8(a) and 8(b)]. This is possible because in those cases there is still a large number of available modes just above the phonon band. Note that the lower limit of the phonon band is given by $\omega=1$; lower frequencies are localized, because they cannot propagate in the system far from the kink, where the spectrum structure is essentially the unperturbed one. This combined effect induces a rapid destabilization of the kink and its trapping. Finally, if the wavelength is further increased, all the first band eventually moves below the phonon band [Fig. 9(d)], and hence long-wavelength emission is strongiy suppressed [Fig. 8(d)], which stabilizes the kink, making possible its propagation. The effect of the shape modes coming from the first band is still revealed by the kink shape oscillations [see Fig. $8(\mathrm{~d})$ ]. We believe that this interpretation clearly explains the mechanisms leading to the appearance of length-scale competition in SG kinks. To seek further evidence, we looked for the approximate value of the potential wave- length at which the last mode in the first band crosses the phonon band. It is shown in Fig. 10 that this happens for a potential wavelength between 5.2 and 5.3. Were our hypothesis true, kinks would not be able to propagate on the former potential and they would be able to do it in the latter one. The numerical simulations shown

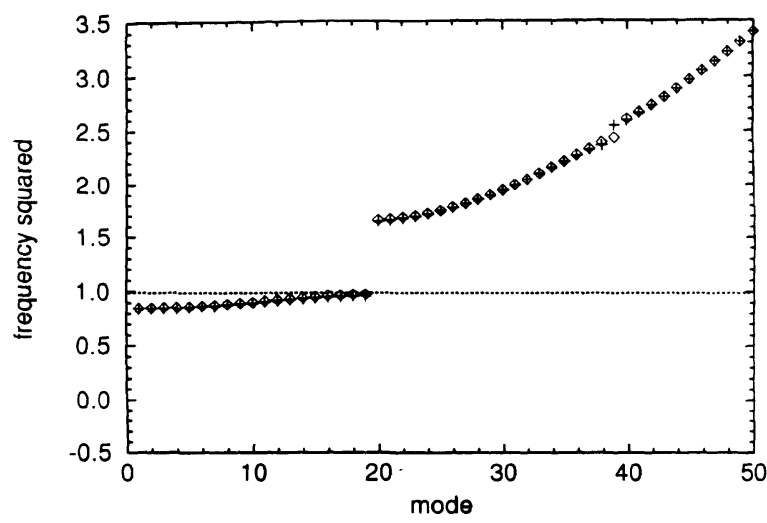

FIG. 10. Spectra for the kink on periodic potentials of wavelengths $5.2(\diamond)$ and $5.3(\times)$. Between these two values, the whole first band of the spectrum occurs below the phonon band. 
in Fig. 11 confirm that this is indeed the case. Interestingly, the radiation is quite different in both cases and, furthermore, for the trapped kink trapping occurs at the second potential well, indicating that the small number of modes available to radiate prevents a very rapid decay of the kink. We thus conclude that our interpretation is indeed correct. As a matter of fact, as this feature of the spectrum will also be present when considering breather propagation (recall that the reason for the appearance of
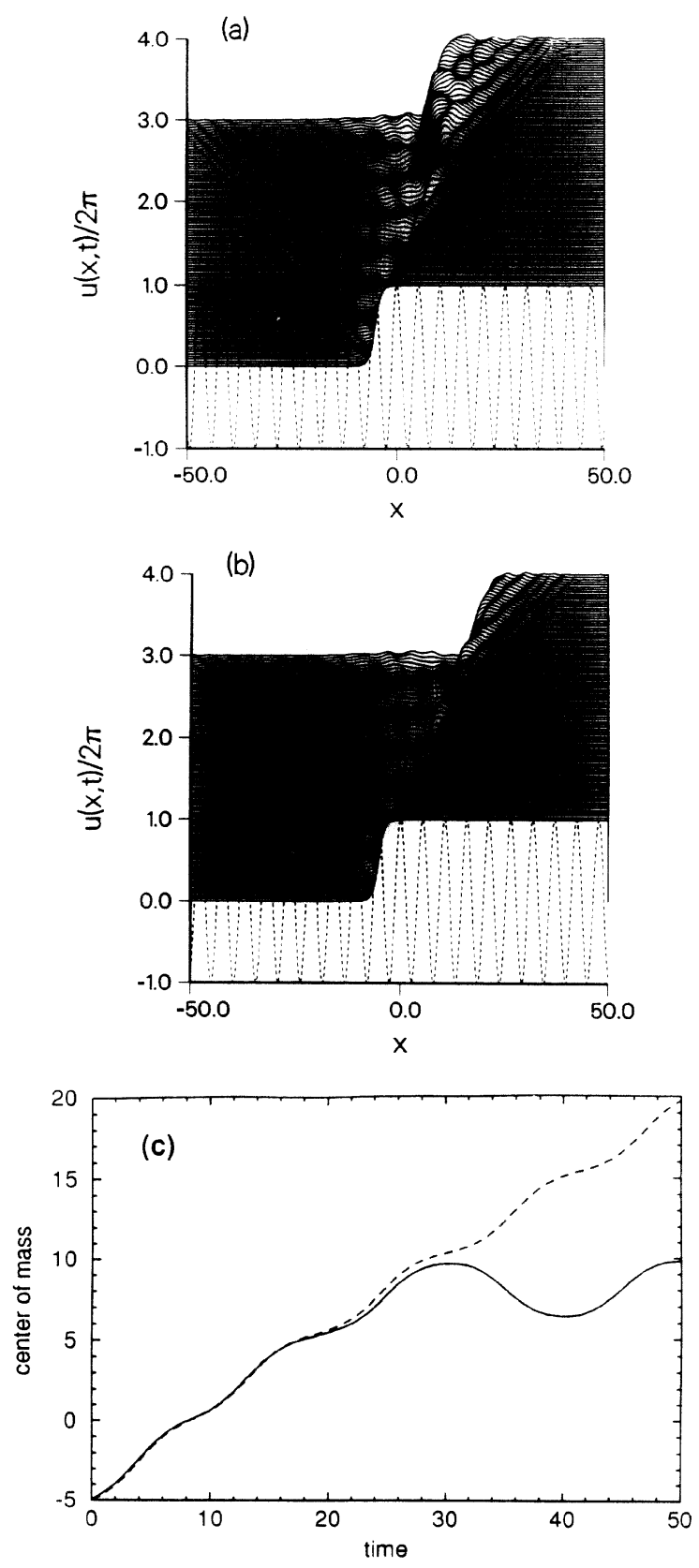

FIG. 11. Numerical verification of the threshold for length-scale competition. Evolution of a kink on a potential of wavelength (a) 5.2 or (b) 5.3. (c) shows the motion of the center of mass in both cases for better comparison; the solid line corresponds to the simulation in (a) and the dashed line to that in (b). the gaps is the perturbating potential acting on the wings of the excitation, and the nature of the center becomes less relevant), our explanation of length-scale competition should also apply to breathers. The results in Ref. [4] are in perfect agreement with what we have described in this section.

\section{CONCLUSIONS}

In this paper, we have studied kink propagation in 1D SG systems with a spatially periodic modulation of its characteristic frequency. We have shown numerically that kinks can propagate steadily and mostly undisturbed, even for large amplitudes of the perturbation. Disagreement with analytical predictions previous to this work is resolved through a new perturbative calculation. By this means, the radiative divergence is shown to be similar to an "infrared" divergence. Our calculation provides us with a good physical understanding of the problem of free kink propagation in the periodically modulated SG system, which had not been obtained from the previous ones, of a more formal character. A comment is in order here, regarding the fact that now the mathematical foundations of perturbation theory for solitons are already established (mostly by pioneering works such as Refs. $[8,9]$ ), the emphasis of that perturbation theory should be focused on their physical implications. Therefore, perturbative calculations in nonlinear equations should be regarded as speculative if they are not verified through numerical simulations and, most importantly, if their physical meaning is not fully established. That is one of the most important points of this work: After an appropriate perturbative calculation, and by carefully considering the dimensions of the problem, we have been able to identify the underlying physical reason for that divergence as a resonance with the lowest, infinite wavelength phonon mode. Moreover, motivated by our perturbative results, we have developed a collective coordinate approach to this problem that describes in a quantitatively correct way the main features of kink propagation, showing that the already known [4] "bare" and "renormalized" particle limits apply also in this case. The collective coordinate equations turn out to predict counterintuitive phenomena whose existence is confirmed by numerical simulations, namely, kink trapping at intermediate points in the potential. This is an important success of the technique. Finally, we have shown that length-scale competition arises unexpectedly in this problem, which afforded us the opportunity to increase our understanding of this ubiquitous phenomenon $[4,6]$. By a detailed linear stability analysis, we have identified the mechanism by which length-scale competition arises as coming from the band structure induced by the perturbation potential. Again, the predictions of our theory have been fully confirmed (quantitatively) by the corresponding numerical simulations.

The global picture that emerges from this work is that, once again, SG kinks behave basically like particles and a collective coordinate approach can be more faithful than complicated perturbative results. Length-scale competi- 
tion phenomena are only relevant in a large amplitude perturbation regime and therefore do not interfere with this picture in most situations. In this respect, it has to be noted that a perturbative calculation describes everything beyond the center of mass dynamics: extended (background) contributions (see, e.g., the third reference in [12]); shape changes localized around the (moving) kink; and the radiation, i.e., emission from the kink. It is crucial to separate and identify these physically different effects if one is to properly understand the dynamics of the considered system. Our results are likely to be general for kink-bearing models in view of the related results of [16] on the spatially periodically perturbed $\phi^{4}$ model. Some remarks are in order regarding this related problem. The same kind of divergence is predicted by a perturbation theory similar to the one used here (see [19] for details on this approach), and again numerical simulations show evidence of the unphysical character of the divergence: It can be seen from Fig. 4 of [16] that as the velocity of a decelerating kink goes through the threshold nothing special occurs. As a matter of fact, most of the reasoning we have used in the present study applies also to that work (with an additional feature coming from the shape mode of the unperturbed $\phi^{4}$ kink), thus reinforcing the generality of our results. On the other hand, the discovery of length-scale competition for SG kinks has allowed us to understand the underlying physical reasons and helped us to gain insight on related results for the SG breather [4]. We believe that the mechanism we have identified in this work is of a very general character and it will be important to have more work on related systems to check our predictions. As a final remark, we want to stress that we have provided a quite thorough description of the features of free kink propagation in a periodically modulated sine-Gordon system and that we have been able to provide a consistent physical framework to understand this "canonical" problem, which will be of help in dealing with more complex situations.

\section{ACKNOWLEDGMENTS}

We are indebted to Rainer Scharf, David Cai, and Maxi San Miguel for enlightening conversations on this research. A.S. is supported by the Ministerio de Educación y Ciencia (Spain) and the Fulbright Fund, by Dirección General de Investigación Científica y Técnica (Spain) through Project No. PB92-0378, and by the European Union (Network on Nonlinear Spatio-Temporal Structures in Semiconductor, Fluids, and Oscillator Ensembles). He also thanks the Los Alamos National Laboratory for warm hospitality and a productive atmosphere. Work at Los Alamos is performed under the auspices of the U.S. Department of Energy.
[1] Disorder and Nonlinearity, edited by A. R. Bishop, D. K. Campbell, and St. Pnevmatikos, Springer Proceedings in Physics Vol. 39 (Springer-Verlag, Berlin, 1989); Nonlinearity with Disorder, edited by F. Kh. Abdullaev, A. R. Bishop, and St. Pnevmatikos, Springer Proceedings in Physics Vol. 67 (Springer-Verlag, Berlin, 1992).

[2] Yu. S. Kivshar and B. A. Malomed, Rev. Mod. Phys. 61, 763 (1989).

[3] A. Sánchez and L. Vázquez, Int. J. Mod. Phys. B 5, 2825 (1991); S. A. Gredeskul and Yu. S. Kivshar, Phys. Rep. 216, 1 (1992).

[4] A. Sánchez, R. Scharf, A. R. Bishop, and L. Vázquez, Phys. Rev. A 45, 6031 (1992).

[5] R. Scharf, Yu. S. Kivshar, A. Sánchez, and A. R. Bishop, Phys. Rev. A 45, R5369 (1992).

[6] R. Scharf and A. R. Bishop, Phys. Rev. E 47, 1375 (1993).

[7] R. Scharf and A. R. Bishop, Phys. Rev. A 46, R2973 (1992).

[8] G. S. Mkrtchyan and V. V. Shmidt, Solid State Commun. 30, 791 (1979).

[9] B. A. Malomed, Phys. Lett. A 144, 351 (1990).

[10] B. A. Malomed and M. I. Tribelsky, Phys. Rev. B 41, 11271 (1990).

[11] A. Sánchez, F. Domínguez-Adame, and A. R. Bishop,
Los Alamos National Laboratory Report No. LA-UR-933740, 1993 (unpublished).

[12] M. B. Fogel, S. E. Trullinger, A. R. Bishop, and J. A. Krumhansl, Phys. Rev. Lett. 24, 1411 (1976); Phys. Rev. B 15, 1578 (1977); M. B. Fogel, S. E. Trullinger, and A. R. Bishop, Phys. Lett. 59A, 81 (1976).

[13] A. Sánchez, L. Vázquez, and V. V. Konotop, Phys. Rev. A 44, 1086 (1991).

[14] W. H. Press, S. A. Teukolsky, W. T. Vetterling, and B. P. Flannery, Numerical Recipes in $C$, 2nd ed. (Cambridge University Press, New York, 1992).

[15] M. Peyrard and M. D. Kruskal, Physica D 14, 88 (1984).

[16] Z. Fei, V. V. Konotop, M. Peyrard, and L. Vázquez, Phys. Rev. E 48, 548 (1993).

[17] R. J. Flesch and S. E. Trullinger, J. Math. Phys. 28, 1619 (1987); A. Galindo and P. Pascual, Quantum Mechanics $I$ (Springer-Verlag, Berlin, 1990).

[18] It is possible to analyze the long wavelength limit of the spectrum analytically by means of an averaging technique, which leads to the result that the minimum frequency is given by $\omega_{\min }^{2}=1-\left(\epsilon^{2} / 2 k^{2}\right)$, in good agreement with our numerical calculation of the spectrum as can be seen from the figures.

[19] V. V. Konotop, A. Sánchez, and L. Vázquez, Phys. Rev. B 44, 2554 (1991). 

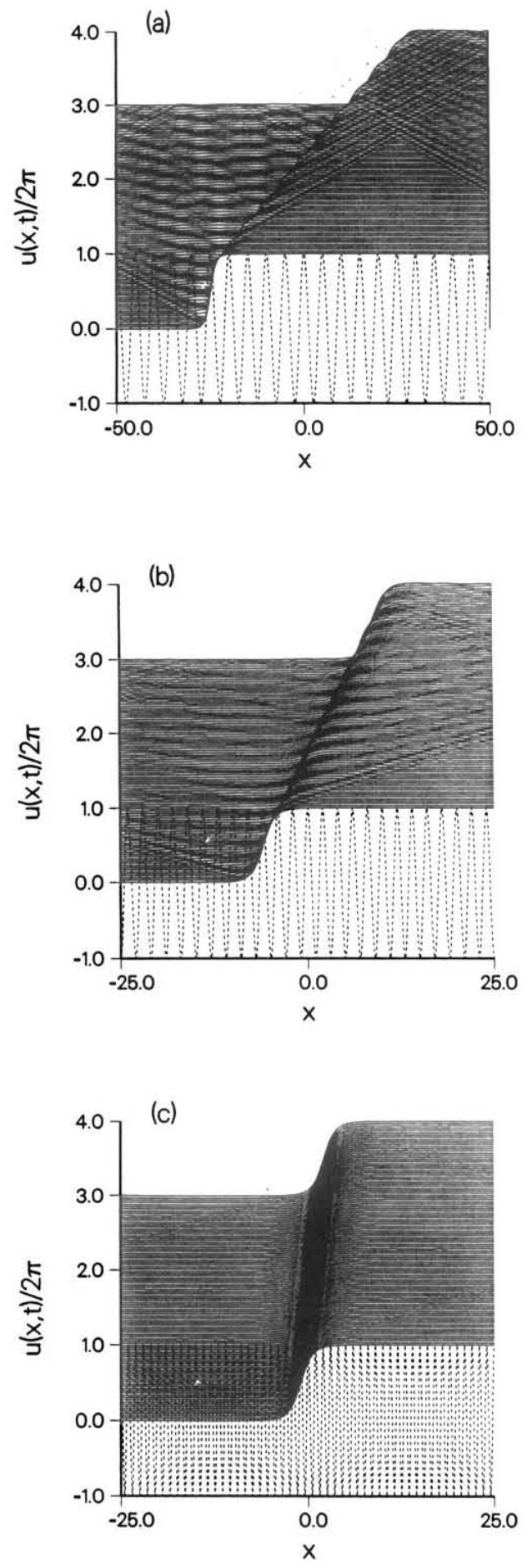

FIG. 1. Absence of radiative divergence for kinks propagating in the spatially periodic SG model. Parameters are (a) $k=2 \pi / 5$, initial velocity $v=v_{\mathrm{thr}}=0.387726 \ldots$; (b) $k=\pi$, initial velocity $v=v_{\mathrm{thr}}=0.091999 \ldots ;$ (c) $k=2 \pi$, initial velocity $v=v_{\mathrm{thr}}=0.024704 \ldots$ [corresponding wavelengths are (a) 5 , (b) 2 , and (c) 1]. In all three cases, $\epsilon=0.4$. The amplitude of the emitted radiation is very small; due to the periodic boundary conditions, it can be seen reentering the simulation interval without any appreciable interaction with the kink. Only half of the simulation interval is shown in plots (b) and (c) to enlarge details. Time increases upwards with final time $t=100$. The potential is indicated by the dashed line (amplitude not to scale). 

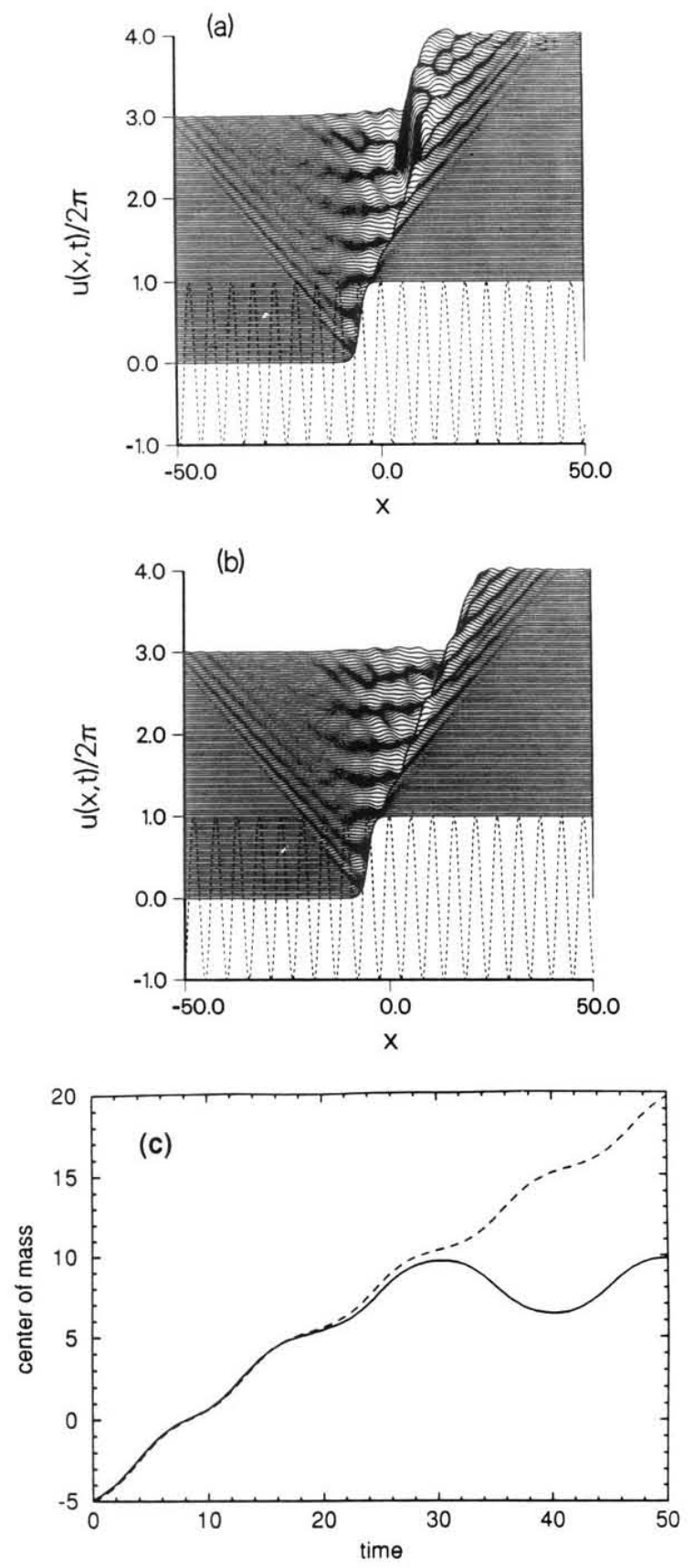

FIG. 11. Numerical verification of the threshold for length-scale competition. Evolution of a kink on a potential of wavelength (a) 5.2 or (b) 5.3. (c) shows the motion of the center of mass in both cases for better comparison; the solid line corresponds to the simulation in (a) and the dashed line to that in (b). 

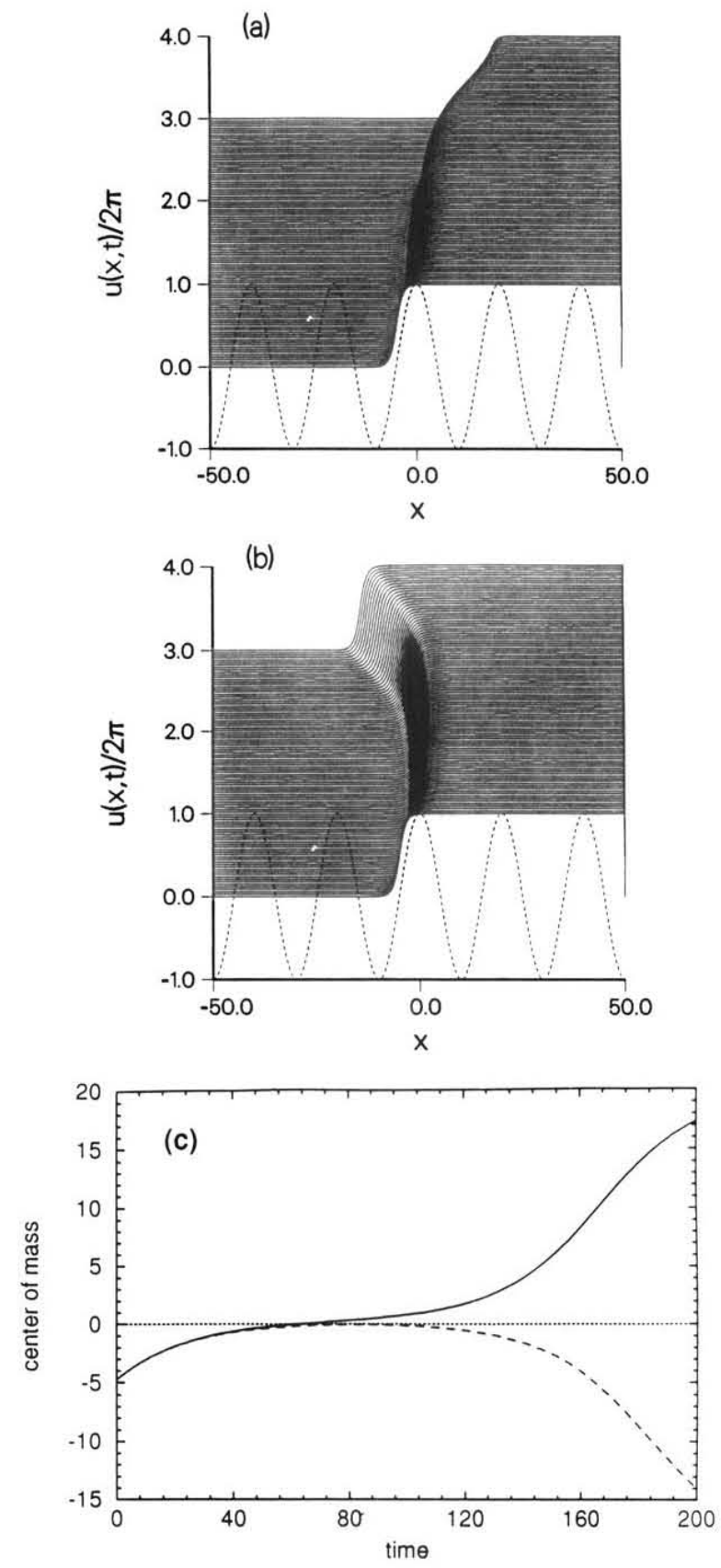

FIG. 4. An example of the verification of the collective coordinate approach predictions. For a kink starting from a midpoint of a potential of wavelength 20 , with velocity 0.2 , the predicted threshold for propagation is $\epsilon_{\mathrm{thr}}=0.0424$. (a) $\epsilon=0.43$ and the kink propagates; (b) $\epsilon=0.435$ and the kink is reflected by the potential maximum; (c) center of mass motion for better comparison of both cases; solid line corresponds to the simulation in (a) and dashed line to that in (b). Final time is $t=200$. Notice the absolute absence of radiation in this phenomenon. 

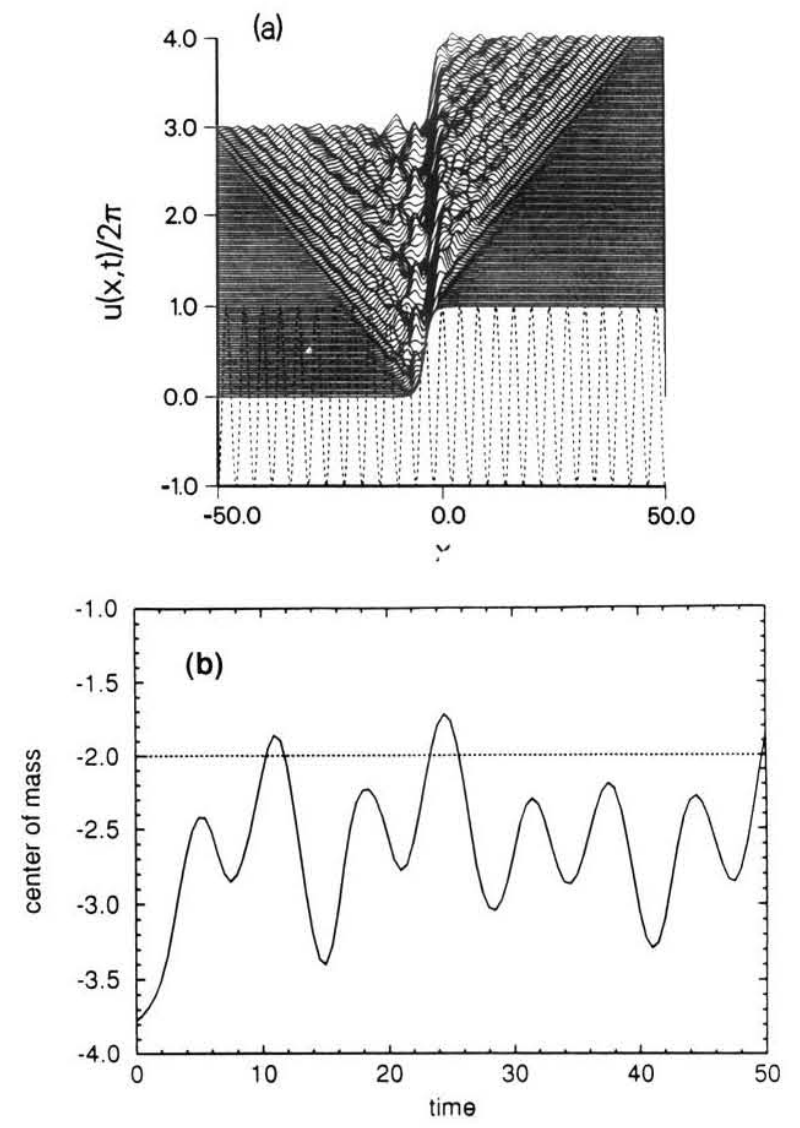

FIG. 6. Trapping of a kink at an intermediate position in the potential instead of at the bottom. $\epsilon=2$ and the kink starts with velocity 0.1 . The amount of emitted radiation is large due to the fast acceleration of the kink in this large perturbation. (a) Time evolution of the kink; (b) time evolution of the center of mass. The neighboring minimum is at $x=-2.0$ (indicated by the dashed line) and the kink oscillates around approximately $x=-2.5$. The final time is $t=50$. 
(a)

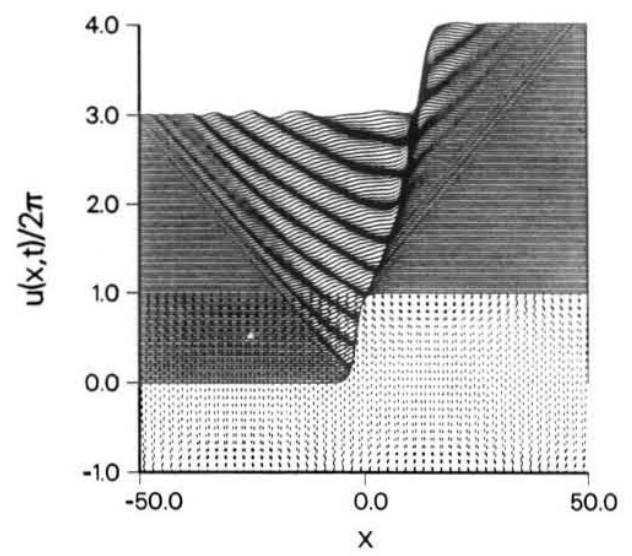

(c)

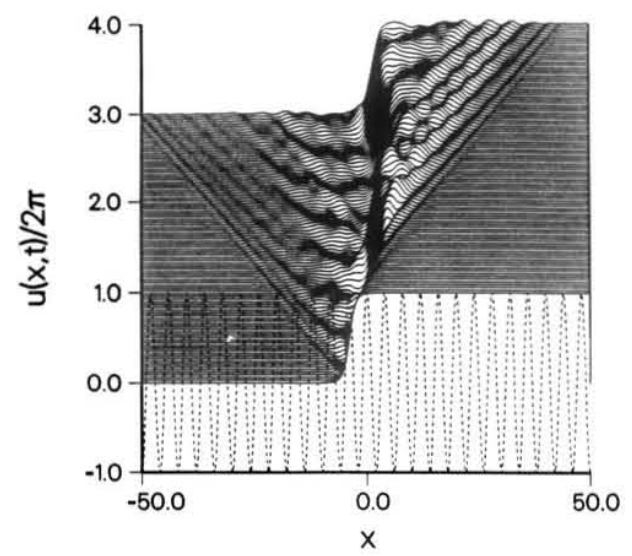

(b)

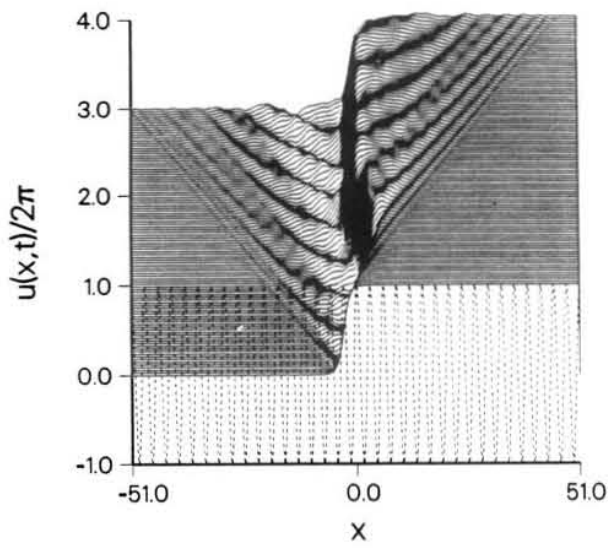

(d)

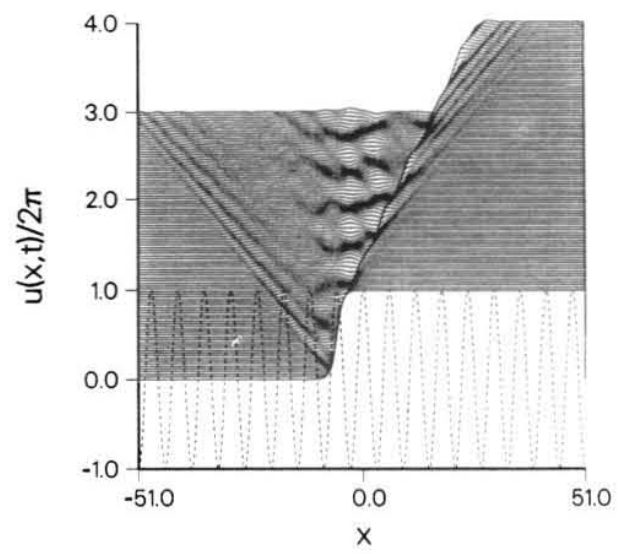

FIG. 8. Time evolution for some of the kinks in Fig. 7. Wavelengths are (a) 2; (b) 3; (c) 4; and (d) 6 . Notice the different kind of radiation emitted in each case. Notice also that the kink in (b) goes back over one maximum of the potential probably due to resonant interaction with radiation left behind. The final time is $t=50$. 